\title{
Preoperative Factors Associated with Remote Postoperative Pain Resolution and Opioid Cessation in a Mixed Surgical Cohort: Post Hoc Analysis of a Perioperative Gabapentin Trial
}

This article was published in the following Dove Press journal:

Journal of Pain Research

Jennifer M Hah, (ID)' Heather Hilmoe, ${ }^{2}$ Peter Schmidt, ${ }^{2}$ Rebecca McCue, ${ }^{2}$ Jodie Trafton, ${ }^{3,4}$ Debra Clay, ${ }^{2}$

Yasamin Sharifzadeh, (iD) ${ }^{2}$

Gabriela Ruchelli, ${ }^{2}$

Tina Hernandez Boussard, (iD ${ }^{5}$

Stuart Goodman, iD ${ }^{6}$

James Huddleston, ${ }^{7}$ William J Maloney, ${ }^{7}$

Frederick M Dirbas, (iD) ${ }^{8}$

Joseph Shrager, ${ }^{9}$ John G Costouros, ${ }^{7}$

Catherine Curtin, (D) ${ }^{10}$

Sean C Mackey, (ID)' lan Carroll, (iD)

'Division of Pain Medicine, Department of Anesthesiology, Perioperative, and Pain Medicine, Stanford University, Stanford, CA, USA; ${ }^{2}$ Stanford Systems Neuroscience and Pain Lab, Stanford University, Stanford, CA, USA; ${ }^{3}$ Department of Psychiatry and Behavioral Sciences, Stanford University, Palo Alto, CA, USA; ${ }^{4}$ VA Program Evaluation and Resource Center, VHA Office of Mental Health

Operations, Palo Alto, CA, USA; ${ }^{5}$ Department of Medicine, Department of Biomedical Data Science, Stanford University, Stanford, CA, USA; ${ }^{6}$ Department of Orthopaedic Surgery and (by Courtesy) Bioengineering, Department of Orthopaedic Surgery, Stanford University, Stanford, CA, USA; ${ }^{7}$ Department of Orthopaedic Surgery, Stanford University, Stanford, CA, USA; ${ }^{8}$ Department of General Surgery, Stanford University, Stanford, CA, USA; ${ }^{9}$ Division of Thoracic Surgery, Stanford University, Stanford, CA, USA; ${ }^{10}$ Division of Hand and Plastic Surgery, Department of Orthopaedic Surgery, Stanford University, Stanford, CA, USA

Correspondence: Jennifer M Hah Division of Pain Medicine, Department of Anesthesiology, Perioperative, and Pain Medicine, Stanford University, 1070

Arastradero Road, Suite 200, Palo Alto, CA 94304, USA

$\mathrm{Tel}+$ I 650-736-94I5

Fax + I 650-725-9642

Email jhah@stanford.edu
Background: Preoperative patient-specific risk factors may elucidate the mechanisms leading to the persistence of pain and opioid use after surgery. This study aimed to determine whether similar or discordant preoperative factors were associated with the duration of postoperative pain and opioid use.

Methods: In this post hoc analysis of a randomized, double-blind, placebo-controlled trial of perioperative gabapentin vs active placebo, 410 patients aged 18-75 years, undergoing diverse operations underwent preoperative assessments of pain, opioid use, substance use, and psychosocial variables. After surgery, a modified Brief Pain Inventory was administered over the phone daily up to 3 months, weekly up to 6 months, and monthly up to 2 years after surgery. Pain and opioid cessation were defined as the first of 5 consecutive days of 0 out of 10 pain or no opioid use, respectively.

Results: Overall, $36.1 \%, 19.8 \%$, and $9.5 \%$ of patients continued to report pain, and $9.5 \%$, $2.4 \%$, and $1.7 \%$ reported continued opioid use at 3, 6, and 12 months after surgery. Preoperative pain at the future surgical site (every 1-point increase in the Numeric Pain Rating Scale; HR 0.93; 95\% CI 0.87-1.00; $P=0.034$ ), trait anxiety (every 10-point increase in the Trait Anxiety Inventory; HR $0.79 ; 95 \%$ CI $0.68-0.92 ; P=0.002)$, and a history of delayed recovery after injury (HR $0.62 ; 95 \%$ CI $0.40-0.96 ; P=0.034$ ) were associated with delayed pain cessation. Preoperative opioid use (HR 0.60; 95\% CI 0.39-0.92; $P=0.020$ ), elevated depressive symptoms (every 5-point increase in the Beck Depression Inventory-II score; HR $0.88 ; 95 \%$ CI $0.80-0.98 ; P=0.017$ ), and preoperative pain outside of the surgical site (HR $0.94 ; 95 \%$ CI $0.89-1.00 ; P=0.046)$ were associated with delayed opioid cessation, while perioperative gabapentin promoted opioid cessation (HR 1.37; 95\% CI 1.06-1.77; $P=0.016$ ). Conclusion: Separate risk factors for prolonged post-surgical pain and opioid use indicate that preoperative risk stratification for each outcome may identify patients needing personalized care to augment universal protocols for perioperative pain management and conservative opioid prescribing to improve long-term outcomes.

Keywords: persistent postsurgical pain, persistent postoperative opioid use, chronic opioid use after surgery, chronic pain after surgery

\section{Introduction}

Surgery presents a natural model for the transition from acute to chronic pain. Examination of pre-existing patient-specific risk factors may elucidate the mechanisms leading to the transition to chronic pain. The high incidence of persistent post-surgical 
pain (PPSP) across a diverse array of operations, ${ }^{1}$ makes examination of postoperative pain an attractive model of pain chronification.

Persistent postoperative opioid use (PPOU) often cooccurs with the development of PPSP as opioids are commonly prescribed for postoperative pain management. ${ }^{2-7}$ The majority of discharged patients prescribed opioids have undergone a surgical procedure. ${ }^{8,9}$ The risk of PPOU is increased even among previously opioid naïve patients who receive opioid prescriptions. ${ }^{9,10}$ Furthermore, approximately $6 \%$ of patients will develop new persistent opioid use regardless of whether they underwent a major or minor operation. ${ }^{11}$ Thus, patient-specific factors may drive postoperative opioid use regardless of the specific operation performed.

The post-surgical pain experience embodies much more than just unidimensional pain intensity driving the transition from acute to chronic pain. To date, the epidemiology of PPSP has commonly focused on the prevalence of pain at months to years after surgery, with a general assumption of a gradual decline in pain between measurements. The prevalence of PPSP through this approach ranges from $57 \%$ at $7-12$ months to $21 \%$ 6-7 years after surgery. ${ }^{12}$

Preliminary research using survival analysis highlights both uniform and disparate preoperative patient-specific predictors of postoperative pain resolution and opioid cessation. Higher self-perceived risk of addiction has been associated with prolonged postoperative pain and opioid use, while preoperative opioid use and elevated depressive symptoms have been associated with prolonged postoperative opioid use. ${ }^{13-15}$ Although opioids are prescribed to treat postoperative pain, the factors associated with PPSP and PPOU may not overlap. These disparate predictors of postoperative pain resolution and opioid cessation have important clinical implications as separate preventative interventions targeting specific risk factors may be needed to decrease PPSP and PPOU among high-risk patients. ${ }^{14}$

As a post hoc secondary analysis of a registered randomized controlled trial, we determined preoperative patient-specific factors (eg, psychosocial, pain, medication use, and substance use) associated with time to pain and opioid cessation in a mixed surgical cohort. We considered a broad range of biopsychosocial patient-specific factors to determine whether or not preoperative risk factors for PPSP and PPOU were similar or discordant.

\section{Materials and Methods}

\section{Study Design and Eligible Participants}

Data for this analysis was collected as part of the Stanford Accelerated Recovery Trial (START). ${ }^{14}$ This was a randomized, double-blind, placebo-controlled trial comparing the effect of perioperative gabapentin vs active placebo on postoperative pain resolution and opioid cessation in a mixed surgical cohort at a tertiary academic medical center. Administration of perioperative gabapentin had no effect on postoperative pain resolution, but had a modest, but significant, effect on reducing the duration of opioid use after surgery. Results from the primary analysis have been reported previously, along with the complete study protocol tracking protocol amendments, and trial methodology. ${ }^{14}$

The study protocol was approved by the Stanford IRB, and registered at clinicaltrials.gov (NCT01067144). The surgical team introduced the study to patients, and the research team subsequently contacted participants for screening and enrollment. All participants provided written, informed consent. The primary study was designed to determine whether perioperative gabapentin promoted postoperative pain and opioid cessation in a mixed surgical cohort of patients undergoing thoracotomy, video-assisted thoracoscopic surgery, primary or revision total hip replacement, primary or revision total knee replacement, unilateral or bilateral mastectomy, breast lumpectomy with or without sentinel node biopsy or axillary node dissection, hand surgery, carpal tunnel surgery, knee arthroscopy, shoulder arthroplasty, and shoulder arthroscopy. Exclusion criteria included known kidney disease, current gabapentin or pregabalin use, cognitive impairment, history of excessive sedation or adverse reaction to gabapentin, coexisting chronic pain (severity level of $>4$ of 10 on the numeric rating scale of pain, with 10 being the most severe level) excluding the planned surgical site, conditions precluding postoperative follow-up, suicidality (as assessed by greater than 0 response to question 9 of the BDI-II), pregnancy, ataxia, dizziness, sedation, narrow-angle glaucoma, severe respiratory insufficiency, history of gastric bypass surgery, and obstructive sleep apnea requiring a continuous positive airway pressure device. A total of 1805 patients, aged 18 to 75 years-old, scheduled for an eligible operation were screened, and 422 patients were randomized for the trial from 2010 to 2014 and followed longitudinally for 2 years postoperatively.

\section{Randomization}

A computer-generated randomization list was created using blocked, stratified randomization by operation and 
surgeon with corresponding randomization log sheets provided to the operating room pharmacy. One log sheet was generated per combination of surgeon and operation. The research pharmacist documented the patient's information on a randomization card that was placed in a sealed envelope indicating randomization allocation. Thus, participants, clinicians, and researchers were blinded to group allocation during the trial and completion of statistical analyses.

\section{Intervention}

A total of 208 patients were randomized to receive gabapentin $1200 \mathrm{mg}$ preoperatively, and $600 \mathrm{mg}$ three times daily postoperatively, and 202 patients were randomized to receive active placebo (lorazepam $0.5 \mathrm{mg}$ ) and inactive placebo three times daily postoperatively for 72 hours. Postoperative pain was treated according to usual clinical practice, with no standardization in opioid prescribing.

\section{Assessments}

The pre-surgical questionnaire packet assessed demographics, disability and employment status, substance use, and pain and opioid use with a modified Brief Pain Inventory (BPI). ${ }^{16}$ Specifically, the questions, "In the last 24 hours, have you needed to take your pain medication for any reason other than just pain, for example to help sleep, to reduce anxiety, or to improve mood?"; "In the last 24 hours, has it been necessary for you to take any more pain medication than recommended?"; "In general, over the last 24 hours which of these options best describes how often you have thought about your pain or pain medication? (with possible responses of less than once per hour, several times an hour, every few minutes, and constantly); "Rate the severity of any pain medication side effects you have experienced over the last 24 hours? (rated on a 0 to 10 scale with 0 representing no side effects and 10 the most severe side effects you can imagine, respectively). Past 30-day prescription opioid use was assessed with the question, "How long has it been since you last used opioid medication on a daily basis?" Several author-generated measures were assessed. Self-reported likelihood of developing chronic pain after surgery ("For you specifically, how likely do you think it is that you will develop a new chronic pain problem after surgery?" with 4 responses from "Not at all" to "Very likely"), self-perceived likelihood of chronic pain after injury ("Do you think you are more or less likely to develop chronic pain following injury than the average person?" with 2 possible responses of "More likely" or "Less likely"), self-perceived sensitivity to pain ("How sensitive are you to pain?" with 3 responses from "Less than average" to "More than average"), self-reported prolonged recovery times from past injuries (Answering "Yes" or "No" to the question, "Have you had unexpectedly long recovery times from injuries in the past?"), and selfperceived likelihood of addiction to pain medication after surgery ("How likely do you think it is that you will develop an addiction problem from pain medication you take after surgery?" with 4 responses from 1: "not at all"; 2: "unlikely"; 3: "somewhat likely"; or 4: "very likely") were included. ${ }^{15}$ The Opioid Risk Tool (ORT) was administered to identify patients at risk for opioid-related aberrant behaviors. ${ }^{17}$ Additional assessments included the Marlow-Crowne Social Desirability Scale to assess social desirability bias, ${ }^{18}$ Barratt Impulsivity Scale to measure impulsive personality traits, ${ }^{19}$ Posttraumatic Stress Disorder Checklist-Civilian Version (PCL-C) to measure post-traumatic stress disorder symptoms, ${ }^{20}$ State-Trait Anxiety Inventory to measure current symptoms of anxiety vs a generalized propensity to be anxious, respectively, ${ }^{21}$ Beck Depression Inventory-II (BDIII) to assess the severity of depressive symptoms, ${ }^{22}$ and the Euroqol Visual Analog Scale (VAS), which is a vertical visual analogue scale with values between 100 (best imaginable health) and 0 (worst imaginable health), for patients to provide a global assessment of their health. ${ }^{23}$

After discharge, a modified BPI was administered over the phone to assess pain related to the surgical site, medication use, and pain interference. The modified BPI was assessed daily up to 3 months, weekly up to 6 months, and monthly up to 2 years after surgery. Specifically, patients reported average pain at the surgical site over the last 24 hours, current pain at the surgical site, and worst pain at the surgical site over the last 24 hours during each call. Pain was rated 0 to 10 per the Numeric Pain Rating Scale (NRS). Calls continued until patients reached pain cessation, opioid cessation, and full recovery up to 2 years after surgery. Additional questions included: "Do you consider yourself to have completely recovered from your surgery?; "If you worked before surgery have you returned to work (whether pain or not-any vocational activity)?"; and "If unemployed, retired, or permanently no longer part of the work force before surgery, have you returned to your presurgery level of activity?" (with possible yes or no responses).

\section{Outcomes}

The primary pre-specified outcomes included time to pain resolution (1st of 5 consecutive days of 0 out of 10 
average pain over the last 24 hours at the surgical site on the numeric rating scale of pain) and time to opioid cessation ( 1 st of 5 consecutive days of 0 opioid use over the last 24 hours). Outcomes were assessed daily up to 3 months, weekly up to 6 months, and monthly thereafter; and the assessment interval ranged from 5 days to 5 months. The majority of patients reached pain and opioid cessation before 6 months. The efficacy of gabapentin on time to pain resolution and time to opioid cessation have been reported previously. ${ }^{14}$

\section{Statistical Analysis}

Due to the explorative post hoc analysis, no statistical power calculation was conducted prior to the analysis as the sample size was based on available data. However, post hoc power analyses were conducted using SAS/STAT 14.2 for the Cox proportional hazards model by using the COXREG option. For the time to pain cessation outcome, we analyzed the power of detecting our smallest effect size for baseline pain at the surgical site. With a two-sided alpha $=0.05$, event probability for pain cessation $=0.685$, R-square of the primary outcome predictor (baseline pain at the surgical site) $=0.05$, hazard ratio $=0.93$, standard deviation $=3$, and total sample size of 410 , we had $95 \%$ power. We also conducted post hoc power analyses for the time to opioid cessation outcome by analyzing the power of detecting the smallest effect size for baseline pain outside the surgical site. With a two-sided alpha $=0.05$, event probability for pain cessation $=0.861$, Rsquare of the primary outcome predictor (baseline pain outside the surgical site) $=0.06$, hazard ratio $=0.94$, standard deviation $=2$, and total sample size of 410 , we had $62 \%$ power. Given that the original clinical trial was powered for the time to pain cessation outcome, it is not surprising that we demonstrate slightly less power for the time to opioid cessation outcome. Hypothesis testing of at least one preoperative covariate having a significant effect on time to pain or opioid cessation respectively was two-tailed. Mean (SD) for continuous preoperative variables and number (\%) were calculated for categorical variables. Time to pain resolution and opioid cessation were analyzed using Kaplan Meier analysis and Cox proportional hazards regression. All analyses in Cox regression were stratified by surgery type to eliminate the operation as a confounder, and to identify universal patientspecific factors influencing the rate of pain resolution and opioid cessation across all operations. Thus, comparisons were only made between subjects undergoing the same operation. Right-censoring of data occurred when patients were lost to follow-up, had a second operation, or asked to be withdrawn. Candidate variables were first evaluated by univariate analyses of pain duration and opioid use duration and included: age, gender, race, ethnicity, marital status, employment status, disability status (claim pending), pain at the future site of surgery, pain elsewhere over the entire body, author-generated measures (self-reported likelihood of developing chronic pain after surgery, likelihood of chronic pain after injury, sensitivity to pain, prolonged recovery times from past injuries, and likelihood of addiction), ORT score, Marlowe-Crowne Social Desirability Scale Score, Barratt Impulsivity Scale Score, PCL-C Score, BDI-II Score, State Anxiety Inventory Score, Trait Anxiety Inventory Score, Euroqol VAS, history of illicit drug use, history of alcohol or drug abuse treatment, randomization group (gabapentin vs active placebo), and past 30-day prescription opioid use. For the multivariable model building process, all preoperative factors were assessed as candidates for the final respective multivariable models. SAS version 9.4 (SAS Institute, Cary, N.D.) was used for all analyses. Inclusion in the final multivariable models was determined with the use of Akaike Information Criteria (AIC) to quantify overall model fit. Sensitivity analyses were conducted to rule out the influence of model selection processes. Automated forward, backward, and stepwise model building algorithms were assessed via AIC scores for optimal model fit. The proportional hazards assumption, and confirmation of nonviolation, was tested by linear regression testing the association of Schoenfeld residuals with time. Relevant covariates in the final models were examined with time interaction terms. Linearity assumptions of continuous predictors in the final multivariable models were tested by examining plots of martingale residuals and cumulative sums of martingale residuals as a function of each continuous predictor, and the supremum test.

\section{Results}

\section{Baseline Characteristics}

Patient characteristics are shown in Table 1. Nineteen thousand five hundred and eleven distinct assessments of postoperative pain, medications, and pain interference occurred throughout the trial and all 410 patients were included in the analysis. Median age was 56.8 (SD 11.6) years. Patients reported postoperative pain for a median of 79 (IQ range 36-230) days exceeding the duration of postoperative opioid use for a median of 27 (IQ range 9-55) days. Postoperative pain duration exceeded opioid use in all surgical categories. Preoperative pain intensity at the future surgical site was 
Table I Baseline Preoperative Characteristics of Patients ${ }^{a}$

\begin{tabular}{|c|c|}
\hline Patients (no.) & 410 \\
\hline Age-yr. & $56.8(11.6)$ \\
\hline Male Gender-no. (\%) & $165(40.4)$ \\
\hline \multicolumn{2}{|l|}{ Marital Status- no.(\%) } \\
\hline Never Married & $34(8.5)$ \\
\hline Married & $292(73.0)$ \\
\hline Living with someone & $15(3.8)$ \\
\hline Divorced or separated & $46(11.5)$ \\
\hline Widowed & $13(3.3)$ \\
\hline Disability Claim Pending- no.(\%) & $46(I I .8)$ \\
\hline \multicolumn{2}{|l|}{ Employment Status-no.(\%) } \\
\hline Full-time & $|7|(43.4)$ \\
\hline Part-time & $36(9.1)$ \\
\hline Unemployed & $79(20.1)$ \\
\hline Retired & $108(27.4)$ \\
\hline \multicolumn{2}{|l|}{ Type of Operation- no.(\%) } \\
\hline Thoracotomy & $15(3.7)$ \\
\hline Total Knee Replacement & $151(36.8)$ \\
\hline Total Hip Replacement & $97(23.7)$ \\
\hline Mastectomy & $4 I(10.0)$ \\
\hline Lumpectomy & $30(7.3)$ \\
\hline VATS & $29(7.1)$ \\
\hline Hand Surgery & $24(5.9)$ \\
\hline Carpal Tunnel Surgery & $5(1.2)$ \\
\hline Knee Arthroscopy & $8(2.0)$ \\
\hline Shoulder Surgery & $4(1.0)$ \\
\hline Shoulder Arthroscopy & $6(1.5)$ \\
\hline Baseline pain at surgical site $(0-10)$ & $5(3)$ \\
\hline Baseline pain other than surgical site $(0-10)$ & $2(2)$ \\
\hline \multirow{2}{*}{\multicolumn{2}{|c|}{$\begin{array}{l}\text { Self-Perceived Likelihood of Developing Chronic Pain } \\
\text { After Surgery- no.(\%) }\end{array}$}} \\
\hline & \\
\hline Not at all & $89(22.2)$ \\
\hline Unlikely & $244(60.9)$ \\
\hline Somewhat likely & $58(\mid 4.5)$ \\
\hline Very likely & $10(2.5)$ \\
\hline \multicolumn{2}{|l|}{$\begin{array}{l}\text { Self-Perceived Likelihood of Chronic Pain After } \\
\text { Injury- no. (\%) }\end{array}$} \\
\hline More Likely & $68(17.1)$ \\
\hline Less Likely & $329(82.9)$ \\
\hline \multicolumn{2}{|l|}{ Self-Perceived Sensitivity to Pain- no. (\%) } \\
\hline More than average & $35(8.8)$ \\
\hline Average & $228(57.0)$ \\
\hline Less than average & $137(34.3)$ \\
\hline $\begin{array}{l}\text { Self-Reported Unexpectedly Long Recovery Times } \\
\text { from Past Injuries-no. (\%) }\end{array}$ & $64(16.0)$ \\
\hline Past 30-Day Prescription Opioid Use- no.(\%) & $36(8.8)$ \\
\hline Self-Perceived Likelihood of Addiction to Pain & \\
\hline Medication After Surgery- no.(\%) & \\
\hline
\end{tabular}

(Continued)
Table I (Continued).

\begin{tabular}{|c|c|}
\hline Patients (no.) & 410 \\
\hline Not at all & $206(5 I .4)$ \\
\hline Unlikely & $172(42.9)$ \\
\hline Somewhat likely & $19(4.7)$ \\
\hline Very likely & $4(1.0)$ \\
\hline Opioid Risk Tool Score ${ }^{c}$ & $2(3)$ \\
\hline History of Illicit Drug Use- no.(\%) & $46(I 1.2)$ \\
\hline History of Alcohol or Drug Abuse Treatment- no.(\%) & $20(5.1)$ \\
\hline Marlow-Crowne Social Desirability Scale Score ${ }^{d}$ & 20(6) \\
\hline Barratt Impulsivity Scale Score ${ }^{e}$ & 69(7) \\
\hline PTSD Checklist-Civilian Version (PCL-C) Score ${ }^{f}$ & $25(9)$ \\
\hline State Anxiety Inventory Score ${ }^{g}$ & $35(11)$ \\
\hline Trait Anxiety Inventory Score ${ }^{\mathrm{h}}$ & $33(10)$ \\
\hline Beck Depression Inventory-II Score ${ }^{i}$ & 10(7) \\
\hline Euroqol VAS ${ }^{i}$ & $72(19)$ \\
\hline Postoperative Pain Duration-days ${ }^{b}$ & $79(36-230)$ \\
\hline Postoperative Opioid Use Duration-days ${ }^{b}$ & $27(9-55)$ \\
\hline
\end{tabular}

Notes: ${ }^{a}$ All values presented as mean (SD) unless otherwise noted, percentages do not

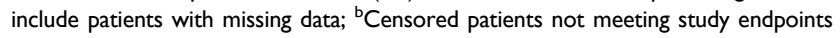
were excluded from calculation of these particular variables, Median (IQ Range); ${ }^{\circ}$ Score range, 0-26, with 0-3 indicating low risk; 4-7 indicating moderate risk, and $\geq 8$ indicating high risk; ${ }^{\mathrm{d}}$ Score range, $0-33$, with $0-8$ indicating low concern for social approval, and 20-33 indicating high concern for social approval; ${ }^{~} S c o r e$ range, 30-120, with higher scores representing greater impulsiveness; 'Score range, 17-85, with increasing scores representing more self-reported posttraumatic stress disorder symptoms; '5Score range, 20-80, with higher scores representing increasing state anxiety or anxiety in response to a specific situation. 395 of 410 participants $(96 \%)$ completed the

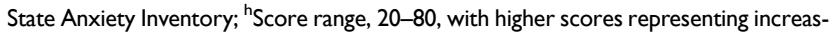
ing trait anxiety or propensity to experience anxiety. 390 of 410 (95\%) participants completed the Trait Anxiety Inventory; 'Score range, 0-63, with 0-13 indicating minimal depression;14-19 mild depression;20-28 moderate depression; and 29-63, severe depression. 398 of 410 (97\%) participants completed the Beck Depression Inventory-II; jScore range for self-assessment of health, 0-100, with 0 representing the worst imaginable health state and 100 representing the best imaginable health state.

higher than that reported outside of the surgical site. The highest proportion of past 30-day prescription opioid use was reported among patients scheduled for shoulder arthroscopy $(17 \%)$ or hand surgery $(13 \%)$. Overall, $68.5 \%$ of patients reached pain cessation two years after surgery with $36.1 \%$ and $19.8 \%$ of patients reporting PPSP 90 and 180 days after surgery, respectively (Table 2). $86.5 \%$ of patients reached opioid cessation two years after surgery with $9.5 \%$ and $2.4 \%$ of patients reporting PPOU 90 and 180 days after surgery, respectively. Survival curves for time to pain and opioid cessation overall are shown in Figure 1.

\section{Univariate Analysis of Postoperative Pain Resolution}

A number of preoperative factors were significantly associated with prolonged pain duration in univariate Cox regression. These included (Table 3): higher preoperative pain at the future surgical site, self-reported prolonged 
Table 2 Time to Pain and Opioid Cessation

\begin{tabular}{|c|c|c|c|}
\hline Endpoint $^{a}$ & $\begin{array}{l}\text { No. (\%) } \\
\text { Reaching } \\
\text { Endpoint }\end{array}$ & $\begin{array}{l}\text { Cumulative No. (\%) } \\
\text { Censored by End of } \\
\text { Interval }\end{array}$ & \\
\hline Time to & & & No. (\%) with \\
\hline Pain & & & Persistent \\
\hline Cessation & & & Pain \\
\hline Day 30 & 81 (19.8) & $32(7.8)$ & $297(72.4)$ \\
\hline Day 60 & $162(39.5)$ & 47 (II.5) & $201(49.0)$ \\
\hline Day 90 & $206(50.2)$ & $56(13.7)$ & $148(36.1)$ \\
\hline Day 180 & $254(62.0)$ & $75(18.3)$ & 81 (19.8) \\
\hline Year I & $277(67.6)$ & 94 (22.9) & $39(9.5)$ \\
\hline Year 2 & $28 I(68.5)$ & $129(3 \mid .5)$ & \\
\hline Time to & & & No. (\%) with \\
\hline Opioid & & & Persistent \\
\hline Cessation & & & Opioid Use \\
\hline Day 30 & $205(50.0)$ & $29(7.0)$ & $176(42.9)$ \\
\hline Day 60 & $296(72.2)$ & $40(9.8)$ & $74(18.0)$ \\
\hline Day 90 & $329(80.2)$ & $42(10.2)$ & $39(9.5)$ \\
\hline Day 180 & $350(85.4)$ & $50(I 2.2)$ & $10(2.4)$ \\
\hline Year I & $353(86.1)$ & $50(12.2)$ & 7 (I.7) \\
\hline Year 2 & $355(86.6)$ & 55 (I3.4) & \\
\hline
\end{tabular}

Note: ${ }^{a} 410$ patients were included in the analysis.

recovery times from past injuries, lower Euroqol VAS score, higher BDI-II score, higher PCL-C score, higher State and Trait Anxiety Score, and patient report of pending disability claim. The proportional hazards assumption was met, and the linearity assumption of significant predictors was met. Of note, a hazard ratio $<1$ represents a reduction in the rate of pain cessation while a hazard ratio $>1$ represents a favorable increase in the rate of pain cessation.

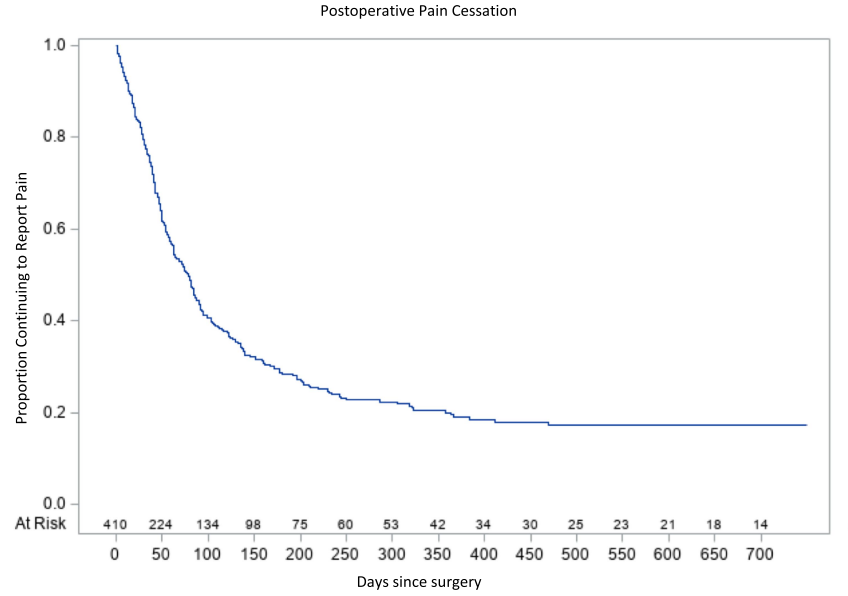

$$
\text { Days since surgery }
$$

Figure I Time to Pain and Opioid Cessation Overall.

\section{Multivariable Analysis of Postoperative Pain Resolution}

The final multivariable model for time to pain cessation was stratified by operation to control for confounding by varying degrees of tissue injury. A number of preoperative factors remained significantly associated with delayed pain resolution across all operations (Table 4). Every 1-point increase in the NRS at the future surgical site was associated with a $7 \%$ reduction in the rate of postoperative pain cessation ( $p$-value=0.034). A selfreported history of prolonged recovery times from past injuries was associated with a $38 \%$ reduction in the rate of postoperative pain cessation ( $p$-value=0.034). Every 10-point increase in the Trait Anxiety Inventory Score was associated with a $21 \%$ reduction in the rate of pain cessation $(p$-value $=0.002)$.

\section{Univariate Analysis of Postoperative Opioid Cessation}

Univariate Cox regression analyses for time to opioid cessation was conducted with stratification by type of operation. A number of preoperative variables were significantly associated with prolonged opioid use (Table 5). Higher preoperative pain, higher self-perceived likelihood of developing chronic pain after surgery, higher self-perceived likelihood of chronic pain after injury, higher BDIII score, higher State Anxiety Inventory score, female gender, randomization to active placebo, and past 30-day prescription opioid use were all significantly associated with prolonged opioid use after surgery.

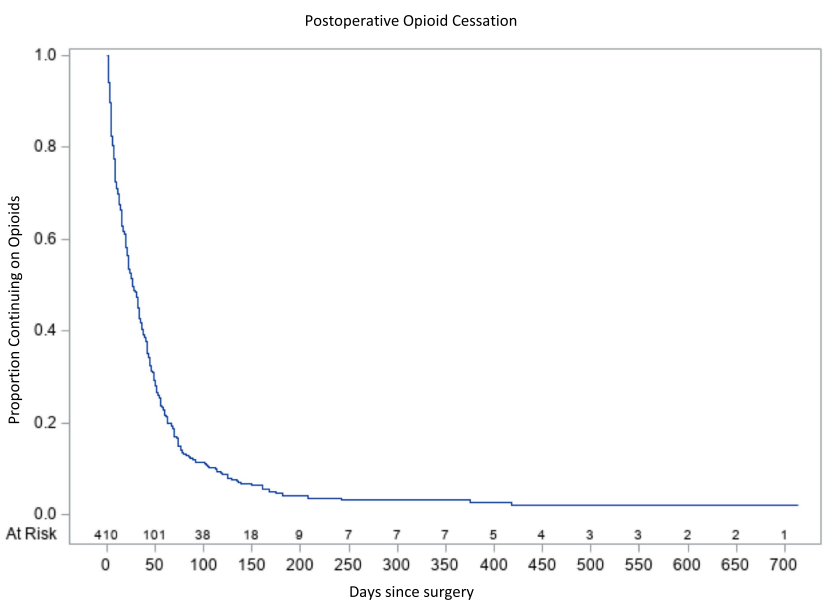


Table 3 Determinants of Pain Cessation Rate: Univariate Analysis

\begin{tabular}{|c|c|c|c|}
\hline Characteristics $^{\mathrm{a}}$ & $\begin{array}{l}\text { Hazard } \\
\text { Ratio }\end{array}$ & $\begin{array}{l}\text { Hazard Ratio } \\
95 \% \\
\text { Confidence } \\
\text { Interval }\end{array}$ & $p$-value \\
\hline $\begin{array}{l}\text { Baseline pain at surgical } \\
\text { site }(0-10)^{b}\end{array}$ & 0.92 & $(0.87-0.98)$ & 0.008 \\
\hline $\begin{array}{l}\text { Self-reported } \\
\text { unexpectedly long } \\
\text { recovery times from past } \\
\text { injuries }\end{array}$ & 0.65 & $(0.45-0.94)$ & 0.021 \\
\hline Euroqol VAS ${ }^{c}$ & 1.10 & $(1.02-1.17)$ & 0.009 \\
\hline $\begin{array}{l}\text { Beck Depression } \\
\text { Inventory-II Score }\end{array}$ & 0.82 & $(0.74-0.91)$ & 0.0002 \\
\hline $\begin{array}{l}\text { PTSD Checklist-Civilian } \\
\text { Version (PCL-C) Score }\end{array}$ & 0.84 & $(0.73-0.96)$ & 0.011 \\
\hline $\begin{array}{l}\text { State Anxiety Inventory } \\
\text { Score }\end{array}$ & 0.81 & $(0.72-0.91)$ & 0.0005 \\
\hline $\begin{array}{l}\text { Trait Anxiety Inventory } \\
\text { Score }\end{array}$ & 0.81 & $(0.7 I-0.92)$ & 0.001 \\
\hline Disability claim pending & 0.58 & $(0.38-0.88)$ & 0.011 \\
\hline
\end{tabular}

Notes: ${ }^{2} \mathrm{Hazard}$ Ratio units represent the minimal clinically important difference for a given continuous variable; ${ }^{b}$ Every I-point increase in the Numeric Pain Rating Scale; 'Every 10-point increased in the Euroqol VAS; ${ }^{\mathrm{d} E v e r y ~ 5-p o i n t ~ i n c r e a s e ~ i n ~ t h e ~}$

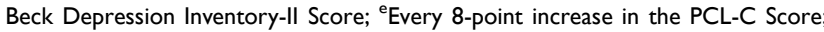
${ }^{f}$ Every 10-point increase in the State Anxiety Inventory Score; ${ }^{8}$ Every 10-point increase in the Trait Anxiety Inventory Score.

\section{Multivariable Analysis of Postoperative Opioid Cessation}

After stratification by type of operation, the final multivariable model for time to opioid cessation included several significant preoperative factors associated with delayed opioid cessation across all surgery types (Table 6). Past 30-day prescription opioid use before surgery was associated with a $40 \%$ reduction in the rate of postoperative opioid cessation ( $p$-value $=0.020$ ). Every 5 -point increase in the BDI-II score was associated with
Table 5 Determinants of Opioid Cessation Rate: Univariate Analysis

\begin{tabular}{|c|c|c|c|}
\hline Characteristics $^{\mathbf{a}}$ & $\begin{array}{l}\text { Hazard } \\
\text { Ratio }\end{array}$ & $\begin{array}{l}\text { Hazard Ratio } \\
\text { 95\% Confidence } \\
\text { Interval }\end{array}$ & $p$-value \\
\hline $\begin{array}{l}\text { Baseline pain at surgical site } \\
(0-10)^{\mathrm{b}}\end{array}$ & 0.93 & $(0.88-0.98)$ & 0.008 \\
\hline $\begin{array}{l}\text { Self-Perceived Likelihood of } \\
\text { Developing Chronic Pain } \\
\text { After Surgery }\end{array}$ & 0.042 & & \\
\hline $\begin{array}{l}\text { Not at all-Reference } \\
\text { Unlikely } \\
\text { Somewhat likely } \\
\text { Very likely-Reference }\end{array}$ & $\begin{array}{l}1.04 \\
0.74 \\
0.44\end{array}$ & $\begin{array}{l}(0.79-1.37) \\
(0.51-1.07) \\
(0.19-1.04)\end{array}$ & \\
\hline $\begin{array}{l}\text { Self-Perceived Likelihood of } \\
\text { Chronic Pain After Injury } \\
\text { More Likely } \\
\text { Less Likely-Reference } \\
\text { Beck Depression } \\
\text { Inventory-II Score } \\
\text { State Anxiety Inventory } \\
\text { Score }^{\text {d }} \\
\text { Female Gender }^{\text {fon }}\end{array}$ & $\begin{array}{l}0.7 \\
0.86 \\
0.89 \\
0.76\end{array}$ & $\begin{array}{l}(0.52-0.94) \\
(0.78-0.94) \\
(0.81-0.99) \\
(0.60-0.96)\end{array}$ & $\begin{array}{l}0.019 \\
0.0006 \\
0.028 \\
0.024\end{array}$ \\
\hline $\begin{array}{l}\text { Treatment Group } \\
\text { Gabapentin } \\
\text { Placebo-Reference } \\
\text { Past 30-Day Prescription } \\
\text { Opioid Use }\end{array}$ & 1.24 & $\begin{array}{l}(1.00-1.54) \\
(0.35-0.79)\end{array}$ & 0.002 \\
\hline
\end{tabular}

Notes: a Hazard Ratio units represent the minimal clinically important difference for a given continuous variable; 'bvery I-point increase in the Numeric Pain Rating Scale; 'Every 5-point increase in the Beck Depression Inventory-II Score; 'Dvery 10point increase in the State Anxiety Inventory Score.

an $12 \%$ decrease in the rate of postoperative opioid cessation ( $p$-value $=0.017)$. Every 1-point increase in the NRS outside the future surgical site was associated with a $6 \%$ reduction in the rate of postoperative pain cessation ( $p$-value $=0.046$ ). Randomization to perioperative gabapentin treatment, after adjusting for these significant preoperative factors, resulted in a $37 \%$ increase in the rate of opioid cessation $(p$-value $=0.016)$.

Table 4 Determinants of Pain Cessation Rate: Multivariable Analysis

\begin{tabular}{|l|l|l|l|}
\hline Characteristics $^{\mathrm{a}}$ & Hazard Ratio & Hazard Ratio 95\% Confidence Interval & p-value \\
\hline${\text { Baseline pain at surgical site }(0-10)^{\mathrm{b}}}^{\mathrm{b}}$ & 0.93 & $(0.87-1.00)$ & 0.034 \\
Self-reported unexpectedly long recovery times from past injuries & 0.62 & $(0.40-0.96)$ & 0.034 \\
Trait Anxiety Inventory Score $^{\mathrm{c}}$ & 0.79 & $(0.68-0.92)$ & 0.002 \\
\hline
\end{tabular}

Notes: ${ }^{a} \mathrm{Hazard}$ Ratio units represent the minimal clinically important difference for a given continuous variable. Results are stratified by operation; ${ }^{\mathrm{b}}$ Every I-point increase in the Numeric Pain Rating Scale; 'Every 10-point increase in the Trait Anxiety Inventory Score. 
Table 6 Determinants of Opioid Cessation Rate: Multivariable Analysis

\begin{tabular}{|l|l|l|l|}
\hline Characteristics $^{\mathbf{a}}$ & $\begin{array}{l}\text { Hazard } \\
\text { Ratio }\end{array}$ & $\begin{array}{l}\text { Hazard Ratio 95\% } \\
\text { Confidence Interval }\end{array}$ & $p$-value \\
\hline $\begin{array}{l}\text { Past 30-Day Prescription } \\
\text { Opioid Use } \\
\text { Beck Depression } \\
\text { Inventory-II Score }\end{array}$ & 0.60 & $(0.39-0.92)$ & 0.020 \\
\hline $\begin{array}{l}\text { Treatment Group } \\
\text { Gabapentin } \\
\text { Placebo-Reference } \\
\text { Baseline pain other } \\
\text { than surgical site (0-10) }\end{array}$ & 0.98 & $(0.80-0.98)$ & 0.017 \\
\hline
\end{tabular}

Notes: ${ }^{a}$ Hazard Ratio units represent the minimal clinically important difference for a given continuous variable. Results are stratified by operation; 'bvery 5-point increase in the Beck Depression Inventory-II Score; 'Every I-point increase in the Numeric Pain Rating Scale.

\section{Post Hoc Sensitivity Analysis}

As additional sensitivity analyses, we included the type of operation as a covariate in each final model. Then, we evaluated the type of operation as an effect modifier of the association of baseline variables that remained significant in the multivariable models (see Supplement). No significant interaction effects were noted between any of the predictors of time to pain cessation and operation type (Table S2). This further indicates that increased baseline pain at the future surgical site, self-reported unexpectedly long recovery times from past injuries, and elevated trait anxiety are significant, independent predictors of delayed pain resolution after surgery. After adding the type of operation to the final multivariable model for time to opioid cessation, past 30-day prescription opioid use and the BDI-II score remained significant predictors (Table

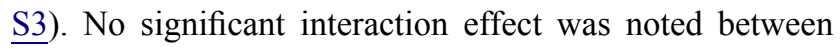
preoperative BDI-II score and type of operation (Table

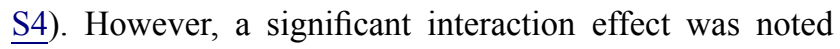
between preoperative past 30-day prescription opioid use and type of operation (Table S5).

\section{Discussion}

In this secondary analysis of a trial of 410 adults receiving either perioperative gabapentin or active placebo, we identified disparate factors associated with prolonged postoperative pain and opioid use. Regardless of the operation, patients with higher levels of preoperative pain at the future surgical site, delayed recovery after past injuries, and elevated anxiety were more likely to experience PPSP. Patients using prescription opioids prior to surgery, with elevated depressive symptoms, higher levels of overall preoperative pain, and those randomized to receive placebo were more likely to develop PPOU. Thus, the clinical trial assignment to gabapentin or active-placebo (lorazepam) had a significant influence on time to opioid cessation, but not on time to pain resolution or PPSP. Opioid cessation occurred before pain cessation across operations. As opioids are overtly prescribed to treat postoperative pain, an assumption may be that the same preoperative risk factors exist for PPSP and PPOU. However, our findings highlight that preoperative determinants of these important postoperative outcomes do not necessarily overlap. An important caveat for our study findings is the wide range of exclusion criteria including known kidney disease, coexisting chronic pain, suicidality, and obstructive sleep apnea requiring a continuous positive airway pressure device used for clinical trial enrollment. Future work to replicate these findings among a wider range of patients with pre-existing chronic pain and chronic medical comorbidities are needed. Thus, preoperative interventions to reduce the risk of PPSP may not passively reduce PPOU, and patients at-risk for PPSP are not necessarily simultaneously at-risk for PPOU at any given time.

Preoperative interventions should be targeted to patients with preoperative risk factors for PPSP and PPOU. Our findings suggest behavioral interventions to reduce anxiety are more likely to promote pain rather than opioid cessation. Antidepressants and cognitive behavioral therapy to reduce depressive symptoms are more likely to promote opioid cessation. Interventions to reduce pain at the future surgical site are more likely to reduce the incidence of PPSP rather than general reductions in total body pain. For example, preoperative targeted genicular nerve denervation for knee osteoarthritis rather than initiation of pain medication may be a more effective strategy for preventing PPSP after total knee arthroplasty. Future research will need to consider critical barriers to implementation of preoperative interventions. Regardless of the whether the focus is behavioral treatments, medications, or interventions, advance planning and treatment pathways must be considered to allow for treatment efficacy prior to surgery. Our work has important implications for preoperative risk stratification, early identification of vulnerable surgical cohorts, and preoperative personalized intervention to complement universal protocols for postoperative pain management and conservative opioid prescribing. 
Prior research examining postoperative pain resolution and opioid cessation describe both uniform and disparate preoperative patient-specific risk factors. Among 107 patients undergoing a variety of operations, greater preoperative self-perceived risk of addiction, and lower anxiety sensitivity was associated with a slower rate of postoperative pain resolution. ${ }^{13}$ Preoperative prescribed opioid use and depressive symptoms were associated with PPOU concordant with our study findings. ${ }^{15}$ This prior study found an association between elevated self-perceived risk of addiction and PPOU, which was not the case in the current study. In patients undergoing lower-extremity joint arthroplasty, higher preoperative opioid use was one of several factors associated with elevated inpatient total postoperative opioid consumption. ${ }^{24}$ We extend these findings by showing the importance of preoperative opioid use in determining remote postoperative opioid use trajectories long after hospital discharge.

In a meta-analysis of 29,993 patients who had undergone total knee arthroplasty, preoperative pain most commonly demonstrated a significant relationship with PPSP. ${ }^{25}$ Our findings emphasize the importance of pain at the future surgical site influencing PPSP. Nociceptor sensitization at the future operative site and subsequent structural changes in the CNS may predispose patients to PPSP. ${ }^{26}$ Surgery results in nociceptive pain from incision, inflammatory pain from release of inflammatory mediators which lower nociceptor thresholds, and neuropathic pain from nerve injury. ${ }^{27}$ Patients with pre-existing nociceptor sensitization at the future surgical site are more likely to experience PPSP.

In a prospective cohort of 300 patients undergoing primary unilateral total knee arthroplasty, preoperative pain intensity, expected pain, and trait anxiety were associated with PPSP. ${ }^{28}$ Similarly, elevated anxiety, rather than depressive symptoms, were associated with PPSP up to 2 years after cardiac surgery. ${ }^{29}$ Our findings expand on prior work by demonstrating an association of trait anxiety with PPSP in a diverse surgical cohort. Among 1,000 women undergoing breast cancer surgery, preoperative state anxiety was associated with preoperative experimental pain sensitivity. ${ }^{30}$ However, no differences in acute postoperative pain intensity were noted based on state anxiety scores. That study did not simultaneously examine trait anxiety. ${ }^{30}$ In patients undergoing total knee arthroplasty or breast cancer surgery, elevated preoperative state rather than trait anxiety was associated with increased postoperative pain intensity $(\geq 3 / 10)$ at 3 months. ${ }^{31}$ As $27 \%$ of patients initially consented did not reach the endpoint, the study may have been biased by the arbitrary endpoint. Also, trait anxiety was assessed 1 month prior, and state anxiety assessed 1 day prior to surgery. This lag time in assessments, may have affected the results. Across a broader range of operations, we demonstrate the importance of trait anxiety in predicting PPOU.

Patients using prescription opioids prior to surgery represent a vulnerable cohort not only at-risk for PPOU, but also at increased risk for mortality, ${ }^{32}$ morbidity, ${ }^{32-35}$ and postoperative healthcare utilization. ${ }^{33,36}$ Prior research demonstrates the association of preoperative opioid use with prolonged postoperative opioid use, and more opioid refills after surgery. ${ }^{37} 64-77 \%$ of chronic opioid users prior to surgery continue chronic opioid use after surgery,38,39 and research highlights preoperative opioid use as an important risk factor for PPOU. ${ }^{38-42}$ Our data add to this body of research by demonstrating the significant association of preoperative opioid use with delayed postoperative opioid cessation compared to demographics, disability status, substance use, and a number of psychological variables.

The association between elevated preoperative depressive symptoms and development of PPOU has been previously reported among patients undergoing lumbar fusion. ${ }^{41}$ Similarly, depression was a risk factor for initiating chronic opioid use after total hip arthroplasty. ${ }^{43}$ Preoperative depressive symptoms may lead to PPOU as opioids blunt the negative emotional responses to the emotional challenges of postoperative recovery, and opioid cessation precipitates mood impairment in these vulnerable patients. The mechanisms by which pre-existing depressive symptoms promote opioid use following injury without prolonging pain are unclear. The most straightforward explanation is that for any given duration of pain, patients delay the transition off opioids to a degree proportional to negative mood symptoms. Among patients receiving chronic opioid therapy for non-cancer pain, depressive symptoms were significantly associated with two forms of self-reported prescription opioid misuse. ${ }^{44}$ Patients with depressive symptoms were more likely to self-report taking more opioids than prescribed, and using opioids for non-pain symptoms such as stress or sleep. ${ }^{44}$ Depressive symptoms may perpetuate postoperative opioid use by promoting opioid misuse and the development of opioid use disorder. The interaction between selective serotonin reupdate inhibitor (SSRI) antidepressants and prodrug prescription opioids provides alternative explanation. SSRI antidepressants inhibit the CYP-2D6 liver enzyme activity required for 
prodrug prescription opioids such as hydrocodone to provide analgesia. Patients simultaneously prescribed SSRI antidepressants and prodrug opioids exhibit worse postoperative pain control at discharge, 3 and 8 weeks after surgery. ${ }^{45}$ However, patients in this clinical trial generally reported minimal or mild depressive symptoms and it is unlikely that all patients were prescribed both SSRI antidepressants and prodrug opioids after surgery.

As preoperative pain outside the future surgical site is a risk factor for prolonged opioid use, patients may use opioids prescribed after surgery to treat overall body pain, and preoperative interventions to optimize pain management may ultimately promote postoperative opioid cessation. A variety of preoperative pain conditions including back pain, neck pain, arthritis, and centralized pain have been reported as risk factors for PPOU. ${ }^{11}$ Thus, preoperative pain intensity outside the future surgical site may be a proxy for the severity and cumulative effect of chronic pain conditions on PPOU.

\section{Strengths and Limitations}

Patients with moderate to severe preoperative pain outside of the surgical site ( $>4$ of 10 on the NRS of pain) and suicidality were excluded. Also, 4 operations included less than 10 participants decreasing study power after stratification by operation. Although sex differences in pain are well described, the risk of female gender on PPSP and PPOU is less clear. ${ }^{46}$ When examining specific operations, gender is more often not associated with postoperative pain. $^{47}$ Age and preoperative chronic pain have been reported as major confounders for sex differences observed in postoperative pain. ${ }^{46}$ As preoperative pain intensity was found to be a significant predictor of PPSP, this may have negated the effects of gender on PPSP. Although higher acute postoperative pain scores are reported among women in the first 2 weeks after total knee arthroplasty, sex differences were no longer evident at 6 weeks. ${ }^{48}$ This may explain why we did not find an association of gender with time to remote pain or opioid cessation. The majority of patients receiving lumpectomy and mastectomy were female. Stratification by type of operation may have reduced the overall effect of gender on pain and opioid trajectories. Despite these limitations, as we have replicated prior findings of the association of preoperative opioid use and elevated depressive symptoms with prolonged postoperative opioid use, it is likely that our study findings are still generalizable.

Our study was powered to test the null hypothesis that gabapentin has no effect on time to pain resolution, and not the current analysis that other preoperative variables influenced time to pain resolution or time to opioid cessation. Because of this, it is possible that we failed to detect real associations between some of our predictor variables and time to pain resolution and time to opioid cessation because of insufficient power.

Overall postoperative pain continues long after opioid cessation. Future research to determine the impact of preoperative pain reduction at the future surgical site, and interventions to reduce anxiety on PPSP, and the efficacy of preoperative interventions to reduce total body pain, depressive symptoms, and preoperative opioid use on PPOU are needed. With the ongoing opioid crisis, increased attention to postoperative opioid prescribing, and legislation limiting the duration of postoperative opioid prescribing, research is urgently needed to provide methods of simultaneously optimizing opioid prescribing and perioperative pain management.

\section{Data Statement}

Due to institutional review board restrictions associated with the trial, participant data is not available to external sources. Proposals to access the de-identified individual participant data that underlie the results reported in this article will be considered 12 months after publication of the Article. Proposals should be directed to the corresponding author (jhah@stanford.edu), with approval by JMH and IC. Requesters will need to sign a data access agreement.

\section{Acknowledgments}

Trial registered with clinicaltrials.gov: NCT01067144.

Principal investigator: Dr. Carroll.

Registration Date: February 11, 2010.

Prior Presentations: None.

\section{Funding}

Dr. Hah (K23DA035302, R01DA045027) and Dr. Carroll (K23DA025152) received funding from the NIH National Institute on Drug Abuse for this project. This project was also funded by the Stanford Department of Anesthesiology, Perioperative, and Pain Medicine, and the Redlich Pain Endowment. The funding organizations had no role in the design and conduct of the study; collection, management, analysis, and interpretation of the data; preparation, review, or approval of the manuscript; and decision to submit the manuscript for publication. 


\section{Disclosure}

Jennifer M Hah reports grants from NIH NIDA, during the conduct of the study. The authors report no other potential conflicts of interest for this work.

\section{References}

1. Steyaert A, Lavand'homme P. Prevention and treatment of chronic postsurgical pain: a narrative review. Drugs. 2018;78(3):339-354. doi:10.1007/s40265-018-0866-X

2. de Beer Jde V, Winemaker MJ, Donnelly GA, et al. Efficacy and safety of controlled-release oxycodone and standard therapies for postoperative pain after knee or hip replacement. Can J Surg. 2005;48(4):277-283.

3. Lindenhovius AL, Helmerhorst GT, Schnellen AC, Vrahas M, Ring D, Kloen P. Differences in prescription of narcotic pain medication after operative treatment of hip and ankle fractures in the United States and The Netherlands. J Trauma. 2009;67(1):160-164 doi:10.1097/TA.0b013e31818c12ee

4. Fischer HB, Simanski CJ. A procedure-specific systematic review and consensus recommendations for analgesia after total hip replacement. Anaesthesia. 2005;60(12):1189-1202. doi:10.1111/j.13652044.2005.04382.x

5. Joshi GP, Rawal N, Kehlet H, et al. Evidence-based management of postoperative pain in adults undergoing open inguinal hernia surgery. Br J Surg. 2012;99(2):168-185. doi:10.1002/bjs.7660

6. Roberts M, Brodribb W, Mitchell G. Reducing the pain: a systematic review of postdischarge analgesia following elective orthopedic surgery. Pain Med. 2012;13(5):711-727. doi:10.1111/j.1526-4637.2012. 01359.x

7. Wunsch H, Wijeysundera DN, Passarella MA, Neuman MD. Opioids prescribed after low-risk surgical procedures in the United States, 2004-2012. JAMA. 2016;315(15):1654-1657. doi:10.1001/jama. 2016.0130

8. Hooten WM, St Sauver JL, McGree ME, Jacobson DJ, Warner DO. Incidence and risk factors for progression from short-term to episodic or long-term opioid prescribing: a population-based study. Mayo Clin Proc. 2015;90(7):850-856. doi:10.1016/j.mayocp.2015.04.012

9. Calcaterra SL, Yamashita TE, Min SJ, Keniston A, Frank JW, Binswanger IA. Opioid prescribing at hospital discharge contributes to chronic opioid use. J Gen Intern Med. 2016;31(5):478-485. doi:10.1007/s11606-015-3539-4

10. Sun EC, Darnall BD, Baker LC, Mackey S. Incidence of and risk factors for chronic opioid use among opioid-naive patients in the postoperative period. JAMA Intern Med. 2016;176(9):1286-1293. doi:10.1001/jamainternmed.2016.3298

11. Brummett CM, Waljee JF, Goesling J, et al. New persistent opioid use after minor and major surgical procedures in US adults. JAMA Surg. 2017;152(6):e170504. doi:10.1001/jamasurg.2017.0504

12. Maguire MF, Ravenscroft A, Beggs D, Duffy JP. A questionnaire study investigating the prevalence of the neuropathic component of chronic pain after thoracic surgery. Eur J Cardiothorac Surg. 2006;29 (5):800-805. doi:10.1016/j.ejcts.2006.02.002

13. Carroll IR, Hah JM, Barelka PL, et al. Pain duration and resolution following surgery: an inception cohort study. Pain Med. 2015;16 (12):2386-2396. doi:10.1111/pme.12842

14. Hah J, Mackey SC, Schmidt P, et al. Effect of perioperative gabapentin on postoperative pain resolution and opioid cessation in a mixed surgical cohort: a randomized clinical trial. JAMA Surg. 2018;153(4):303-311. doi:10.1001/jamasurg.2017.4915

15. Carroll I, Barelka P, Wang CK, et al. A pilot cohort study of the determinants of longitudinal opioid use after surgery. Anesth Analg. 2012;115(3):694-702.
16. Keller S, Bann CM, Dodd SL, Schein J, Mendoza TR, Cleeland CS Validity of the brief pain inventory for use in documenting the outcomes of patients with noncancer pain. Clin J Pain. 2004;20(5):309 318. doi:10.1097/00002508-200409000-00005

17. Webster LR, Webster RM. Predicting aberrant behaviors in opioidtreated patients: preliminary validation of the opioid risk tool. Pain Medicine. 2005;6(6):432-442. doi:10.1111/j.1526-4637.2005.00072.x

18. Crowne DP, Marlowe D. A new scale of social desirability independent of psychopathology. J Consult Psychol. 1960;24:349-354. doi: 10.1037/h0047358

19. Patton JH, Stanford MS, Barratt ES. Factor structure of the Barratt impulsiveness scale. J Clin Psychol. 1995;51(6):768-774.

20. Ruggiero KJ, Del Ben K, Scotti JR, Rabalais AE. Psychometric properties of the PTSD checklist-civilian version. J Trauma Stress. 2003;16(5):495-502. doi:10.1023/A:1025714729117

21. Spielberger CD. State-Trait Anxiety Inventory: A Comprehensive Bibliography. 2nd ed. Palo Alto, CA (577 College Ave., Palo Alto 94306): Consulting Psychologists Press; 1989.

22. Beck AT, Steer RA, Ball R, Ranieri W. Comparison of Beck Depression Inventories -IA and -II in psychiatric outpatients. J Pers Assess. 1996;67(3):588-597. doi:10.1207/s15327752jpa6703_13

23. EuroQol G. EuroQol-a new facility for the measurement of healthrelated quality of life. Health Policy (New York). 1990;16(3):199208.

24. Brummett CM, Janda AM, Schueller CM, et al. Survey criteria for fibromyalgia independently predict increased postoperative opioid consumption after lower-extremity joint arthroplasty: a prospective, observational cohort study. Anesthesiology. 2013;119(6):1434-1443. doi:10.1097/ALN.0b013e3182a8eb1f

25. Lewis GN, Rice DA, McNair PJ, Kluger M. Predictors of persistent pain after total knee arthroplasty: a systematic review and metaanalysis. $\mathrm{Br} J$ Anaesth. 2015;114(4):551-561. doi:10.1093/bja/ aeu441

26. Fregoso G, Wang A, Tseng K, Wang J. Transition from acute to chronic pain: evaluating risk for chronic postsurgical pain. Pain Physician. 2019;22(5):479-488

27. Borsook D, Kussman BD, George E, Becerra LR, Burke DW. Surgically induced neuropathic pain: understanding the perioperative process. Ann Surg. 2013;257(3):403-412. doi:10.1097/SLA.0b013e $3182701 \mathrm{a} 7 \mathrm{~b}$

28. Rice DA, Kluger MT, McNair PJ, et al. Persistent postoperative pain after total knee arthroplasty: a prospective cohort study of potential risk factors. Br J Anaesth. 2018;121(4):804-812. doi:10.1016/j. bja.2018.05.070

29. Page MG, Watt-Watson J, Choiniere M. Do depression and anxiety profiles over time predict persistent post-surgical pain? A study in cardiac surgery patients. Eur J Pain. 2017;21(6):965-976. doi:10. 1002/ejp.998

30. Kaunisto MA, Jokela R, Tallgren M, et al. Pain in 1,000 women treated for breast cancer: a prospective study of pain sensitivity and postoperative pain. Anesthesiology. 2013;119(6):1410-1421. doi:10. 1097/ALN.0000000000000012

31. Masselin-Dubois A, Attal N, Fletcher D, et al. Are psychological predictors of chronic postsurgical pain dependent on the surgical model? A comparison of total knee arthroplasty and breast surgery for cancer. $J$ Pain. 2013;14(8):854-864. doi:10.1016/j.jpain.2013.02.013

32. Barrantes F, Luan FL, Kommareddi M, et al. A history of chronic opioid usage prior to kidney transplantation may be associated with increased mortality risk. Kidney Int. 2013;84(2):390-396. doi:10. 1038/ki.2013.136

33. Cron DC, Englesbe MJ, Bolton CJ, et al. Preoperative opioid use is independently associated with increased costs and worse outcomes after major abdominal surgery. Ann Surg. 2017;265:695-701.

34. Zywiel MG, Stroh DA, Lee SY, Bonutti PM, Mont MA. Chronic opioid use prior to total knee arthroplasty. J Bone Joint Surg Am. 2011;93(21):1988-1993. doi:10.2106/JBJS.J.01473 
35. Shanmugam VK, Couch KS, McNish S, Amdur RL. Relationship between opioid treatment and rate of healing in chronic wounds. Wound Repair Regen. 2017;25:120-130.

36. Rogal S, Mankaney G, Udawatta V, et al. Association between opioid use and readmission following liver transplantation. Clin Transplant. 2016;30(10):1222-1229. doi:10.1111/ctr.12806

37. Waljee JF, Zhong L, Hou H, Sears E, Brummett C, Chung KC. The use of opioid analgesics following common upper extremity surgical procedures: a national, population-based study. Plast Reconstr Surg. 2016;137(2):355e-364e. doi:10.1097/01.prs.0000475788.52446.7b

38. Raebel MA, Newcomer SR, Reifler LM, et al. Chronic use of opioid medications before and after bariatric surgery. JAMA. 2013;310 (13):1369-1376. doi:10.1001/jama.2013.278344

39. Zarling BJ, Yokhana SS, Herzog DT, Markel DC. Preoperative and postoperative opiate use by the arthroplasty patient. J Arthroplasty. 2016;31(10):2081-2084. doi:10.1016/j.arth.2016.03.061

40. Raebel MA, Newcomer SR, Bayliss EA, et al. Chronic opioid use emerging after bariatric surgery. Pharmacoepidemiol Drug Saf. 2014;23(12):1247-1257. doi:10.1002/pds.3625

41. Anderson JT, Haas AR, Percy R, Woods ST, Ahn UM, Ahn NU. Chronic opioid therapy after lumbar fusion surgery for degenerative disc disease in a workers' compensation setting. Spine (Phila Pa 1976). 2015;40 (22):1775-1784. doi:10.1097/BRS.0000000000001054

42. Kulshrestha S, Barrantes F, Samaniego M, Luan FL. Chronic opioid analgesic usage post-kidney transplantation and clinical outcomes. Clin Transplant. 2014;28(9):1041-1046. doi:10.1111/ctr.12414
43. Inacio MC, Hansen C, Pratt NL, Graves SE, Roughead EE. Risk factors for persistent and new chronic opioid use in patients undergoing total hip arthroplasty: a retrospective cohort study. BMJ Open. 2016;6(4):e010664. doi:10.1136/bmjopen-2015-010664

44. Grattan A, Sullivan MD, Saunders KW, Campbell CI, Von Korff MR. Depression and prescription opioid misuse among chronic opioid therapy recipients with no history of substance abuse. Ann Fam Med. 2012;10(4):304-311. doi:10.1370/afm.1371

45. Parthipan A, Banerjee I, Humphreys K, et al. Predicting inadequate postoperative pain management in depressed patients: a machine learning approach. PLoS One. 2019;14(2):e0210575. doi:10.1371/ journal.pone.0210575

46. Zheng H, Schnabel A, Yahiaoui-Doktor M, et al. Age and preoperative pain are major confounders for sex differences in postoperative pain outcome: a prospective database analysis. PLoS One. 2017;12 (6):e0178659. doi:10.1371/journal.pone.0178659

47. Dorow M, Lobner M, Stein J, et al. Risk factors for postoperative pain intensity in patients undergoing lumbar disc surgery: a systematic review. PLoS One. 2017;12(1):e0170303. doi:10.1371/journal. pone. 0170303

48. Nandi M, Schreiber KL, Martel MO, et al. Sex differences in negative affect and postoperative pain in patients undergoing total knee arthroplasty. Biol Sex Differ. 2019;10(1):23. doi:10.1186/s13293-0190237-7

\section{Publish your work in this journal}

The Journal of Pain Research is an international, peer reviewed, open access, online journal that welcomes laboratory and clinical findings in the fields of pain research and the prevention and management of pain. Original research, reviews, symposium reports, hypothesis formation and commentaries are all considered for publication. The manuscript management system is completely online and includes a very quick and fair peer-review system, which is all easy to use. Visit http:// www.dovepress.com/testimonials.php to read real quotes from published authors. 